\title{
ROS1 fusion lung adenosquamous carcinoma patient with short- term clinical benefit after crizotinib treatment: a case report
}

\author{
Yurong Cheng, Jiandong Yang, Daochao Wang, Dong Yan \\ Department of Oncology, Beijing Luhe Hospital, Capital Medical University, Beijing, China \\ Correspondence to: Dong Yan. Department of Oncology, Beijing Luhe Hospital, Capital Medical University, Beijing 101149, China. \\ Email: yd15yt88@163.com.
}

\begin{abstract}
ROS1 rearrangements are validated drivers in non-small cell lung cancer (NSCLC), and occur at an extremely low rate in rare pathological subtypes such as adenosquamous carcinoma (ASC). Crizotinib is known to be effective in patients with ROS1-rearranged NSCLC. However, the efficacy of crizotinib in patients with ROS1-rearranged lung ASC is unknown. Here, we report the case of a 43 -year-old female never-smoker who presented with dry cough for 3 months. The patient was then diagnosed with stage IIIA poorly-differentiated lung ASC with ROS1 rearrangement (CD74-ROS1). Programmed death-ligand 1 (PDL1) expression was high with $50 \%$ in tumor cells of her lung puncture biopsy sample. The patient received albumin-bound paclitaxel and camrelizumab as the first-line treatment and achieved a stable disease (SD) response with progression-free survival (PFS) of 2 months. Subsequently, the patient received crizotinib as the second-line treatment and achieved a partial response (PR) with PFS of 4 months. No gene mutation other than CD74-ROS1 (C6:R34) rearrangement was detected from the lung biopsy sample after crizotinib resistance using a panel covering 520 cancer-related genes. We speculate that crizotinib may have a short duration of efficacy against lung ASC. This is the first case report of response to crizotinib for a lung ASC patient with ROS1 fusion, and may help future targeted therapy investigations and prognostic evaluation for patients with rare pathological subtypes of NSCLC.
\end{abstract}

Keywords: ROS1; crizotinib; lung adenosquamous carcinoma (ASC); case report

Submitted Nov 26, 2021. Accepted for publication Jan 12, 2022.

doi: 10.21037/atm-21-6754

View this article at: https://dx.doi.org/10.21037/atm-21-6754

\section{Introduction}

Non-small cell lung cancer (NSCLC) is a malignant cancer that has the highest mortality rate of all cancers worldwide (1). As a rare subtype of NSCLC, adenosquamous carcinoma (ASC) of the lung is defined as a malignancy containing components of lung adenocarcinoma (ADC) and lung squamous cell carcinoma (SCC) (2). With a frequency of $0.4-4 \%$ in lung cancer, ASC is generally considered to be an aggressive cancer with poor prognosis (2-4). There is no unified standard chemotherapy for ASC, and current treatment options depend on the NSCLC guidelines. Surgical resection is the preferred treatment option for ASC patients. Epidermal growth factor receptor tyrosine kinase inhibitors (EGFR-TKIs), such as gefitinib and erlotinib, may be effective therapeutic strategies for advanced ASC with EGFR mutation (2). However, studies on crizotinib in the treatment of ASC are very limited. Only 1 case of a postoperative ASC patient with $A L K$ rearrangement who benefited from crizotinib treatment after chemotherapy failure has been reported (5). Immune checkpoint blockade therapy is under clinical investigation and may be a potential treatment choice for ASC patients.

The proto-oncogene ROS1, which belongs to the sevenless subfamily of tyrosine kinase insulin receptor genes, has been found to be highly expressed in various tumor cells (TC) (6). The protein it encodes, ROS1, is a receptor tyrosine kinase (RTK), which function as surface receptors for cellular signal transduction and play crucial roles in the proliferation, differentiation, and migration of 
cancer cells (7). ROS1 rearrangement has been identified with an incidence of $0.5-2 \%$ in NSCLC, and mostly occurs in ADC (8). The oncogenic rearrangement commonly results in the fusion of the $3^{\prime}$ end kinase domain of ROS1 and leads to the persistent activation of downstream signaling pathways. Several genes have been distinguished as the fusion partners of ROS1, such as CD74, EZR, CCD6, and TPM3 $(9,10)$. Nowadays, multiple methods have been clinically applied to characterize ROS1-rearranged NSCLC, including next-generation sequencing (NGS), polymerase chain reaction (PCR), fluorescence in situ hybridization (FISH), and immunohistochemistry (IHC) (9).

As an ATP-competitive small-molecule inhibitor of MET/ALK/ROS1, crizotinib has demonstrated an impressive objective response rate (ORR) of $72 \%$, median progressionfree survival (PFS) of 19.2 months, and overall survival of 51.4 months in patients with advanced metastatic ROS1rearranged NSCLC, and has been clinically proved for the treatment of ROS1-rearranged NSCLC $(11,12)$. Since the prevalence of ROS1 fusion in ASC is extremely low, the efficacy of crizotinib in rare pathological subtypes of ROS1fusion NSCLC has rarely been reported. Here, we revealed a case demonstrating the therapeutic effect of crizotinib and checkpoint inhibitor treatment in an ASC patient harboring CD74-ROS1 fusion. We present the following case in accordance with the CARE reporting checklist (available at https://atm.amegroups.com/article/view/10.21037/atm-21$6754 / \mathrm{rc})$.

\section{Case presentation}

A 43-year-old female never-smoker presented with dry cough for 3 months and sought medical advice in December 2019. She had no specific medical history and no family history of cancer. She had an Eastern Cooperative Oncology Group (ECOG) performance status score of 0 . In order to obtain an accurate diagnosis, she went to many hospitals for diagnostic tests. Figure 1 shows the timeline and treatment history of this patient. Computed tomography (CT) scans of the chest showed a space-occupying lesion which was measured $3.3 \mathrm{~cm} \times 3.4 \mathrm{~cm} \times 3.0 \mathrm{~cm}$ in her right upper lobe, while multiple enlarged mediastinal lymph nodes were also observed. Positron emission tomography/CT (PET/CT) showed significant uptake in the lesion and mediastinal lymph nodes. Through IHC of the lung puncture biopsy sample, the patient was diagnosed with poorlydifferentiated $\mathrm{ADC}$ of the right lung (pT2aN2M0, stage IIIA) with focal squamous differentiation (CK7 positive,
CK18 positive, TTF-1 positive, CK5/6 focally positive, $\mathrm{P} 40$ focally positive, P63 focally positive, Napsin A focally and weakly positive). CD74-ROS1 rearrangement was identified by targeted NGS of the biopsy sample, and no other actionable mutation was detected. The tumor mutational burden (TMB) of this biopsy sample was 0.8 mutation/MB. IHC staining of programmed death-ligand 1 (PD-L1) was performed using SP142 antibody, and PD-L1 expression was $50 \%$ in TC and $<1 \%$ in tumor-infiltrating immune cells (IC).

Subsequently, the patient received albumin-bound paclitaxel $260 \mathrm{mg} / \mathrm{m}^{2}$ i.v. and camrelizumab $200 \mathrm{mg}$ i.v. every 3 weeks as the first-line therapy in January 2020, and achieved stable disease (SD) after 1 cycle treatment based on the Response Evaluation Criteria in Solid Tumors (RECIST) 1.1. The radiographic response to each treatment is also shown in Figure 1. However, 2 months later, the disease progressed due to the increase in the size of pulmonary lesion and supraclavicular lymph node metastasis. Therefore, the PFS of albumin-bound paclitaxel plus camrelizumab therapy was 2 months. Subsequently, the patient received crizotinib (250 $\mathrm{mg}$ orally twice daily) as the second-line therapy in March 2020. One month later, in April 2020, CT scans revealed shrunken pulmonary lesions indicating a partial response (PR). Unfortunately, after 4 months of crizotinib therapy, in July 2020, the patient began to experience intermittent dyspnea, and was evaluated as progressive disease (PD) due to the increase in the size of pulmonary lesion, which was measured $6.8 \mathrm{~cm} \times 7.5 \mathrm{~cm}$ $\times 9.0 \mathrm{~cm}$, and had associated liver and brain metastases. Bronchoscopic biopsy was conducted and additional molecular testing was performed after disease progression using a panel of 520 cancer-related genes (OncoScreen Plus ${ }^{\mathrm{TM}}$, Burning Rock Biotech, Guangzhou, China). NGS of the biopsy tissue sample from the primary lung lesion did not show any clinically significant mutations other than CD74-ROS1 (C6:R34) rearrangement (Figure 2A). The TMB of this lung biopsy sample was 0 mutation/MB. PDL1 expression of the lung biopsy tissue was assessed with the 22C3 pharmDx assay, and the tumor proportion score (TPS) of PD-L1 was $80 \%$ (Figure $2 B$ ). Crizotinib was withdrawn, and the patient received pemetrexed $(800 \mathrm{mg}$ $\mathrm{d} 1$ ), carboplatin (400 $\mathrm{mg} \mathrm{d} 1$ ), and bevacizumab (500 mg d1) every 3 weeks in July 2020. She achieved a PR after 2 cycles of therapy. However, 4 months later, her intrapulmonary and liver metastatic lesions progressed. No obvious toxic and side effects were observed during the whole treatment above. After 5 cycles of therapy, the patient switched to 


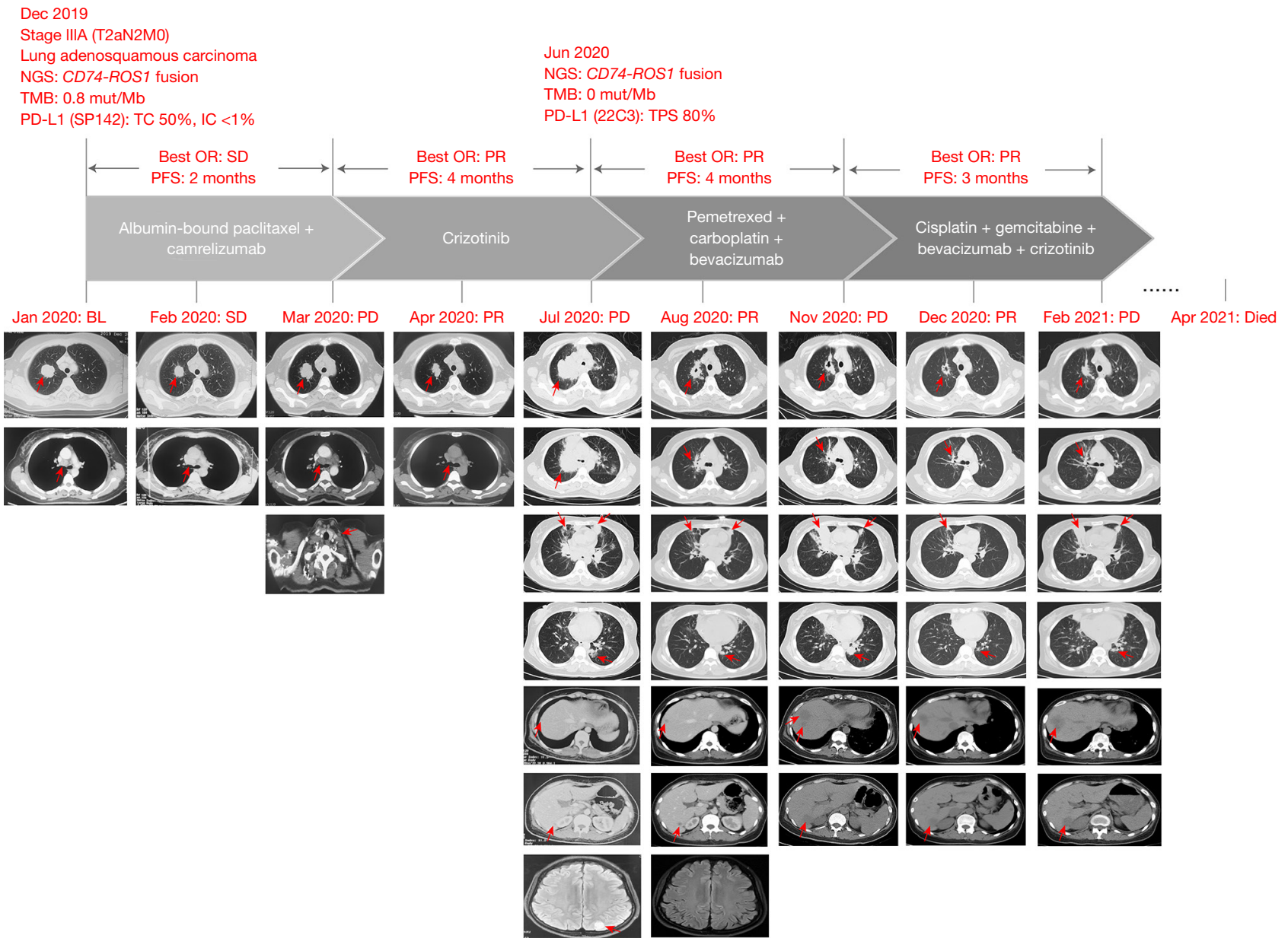

Figure 1 The timeline, treatment history and radiographic response to treatment of the patient. The locations of the lesions were marked with red arrow. Best OR, best objective response; PR, partial response; SD, stable disease; PFS, progression-free survival; NGS, next-generation sequencing; TMB, tumor mutational burden; PD-L1, programmed death-ligand 1; TC, tumor cells; IC, immune cells; TPS, tumor proportion score; BL, baseline.

receive cisplatin (60 $\mathrm{mg} \mathrm{d} 1-2)$, gemcitabine (1.6 g d1), and bevacizumab $(500 \mathrm{mg} \mathrm{d} 1)$ every 3 weeks and crizotinib ( $250 \mathrm{mg}$ orally twice daily) in November 2020. She achieved a PR after 2 cycles of therapy. Then, the disease progressed after 5 cycles of therapy in February 2021. Nausea (grade 1) and bone marrow suppression after chemotherapy were observed during this treatment. The patient's compliance was excellent during the whole treatment. However, the tumors could not be controlled and she eventually died on April $28^{\text {th }}$ of 2021.

All procedures performed in this study were in accordance with the ethical standards of the institutional and/or national research committee(s) and with the Helsinki Declaration (as revised in 2013). Written informed consent was obtained from the patient for publication of this case report and accompanying images. A copy of the written consent is available for review by the editorial office of this journal.

\section{Discussion}

ASC is a special subtype of NSCLC, different from ADC and SCC, with a low incidence, strong aggressiveness, and poor prognosis (2). Crizotinib is an established treatment option for advanced metastatic ALK-positive or ROS1positive NSCLC $(11,13)$. However, studies on crizotinib in the treatment of ASC are very limited. Only 1 ASC patient with $A L K$ rearrangement was reported to have prolonged overall tumor shrinkage lasting 13 months after crizotinib treatment (5). Here, we present the first case demonstrating 

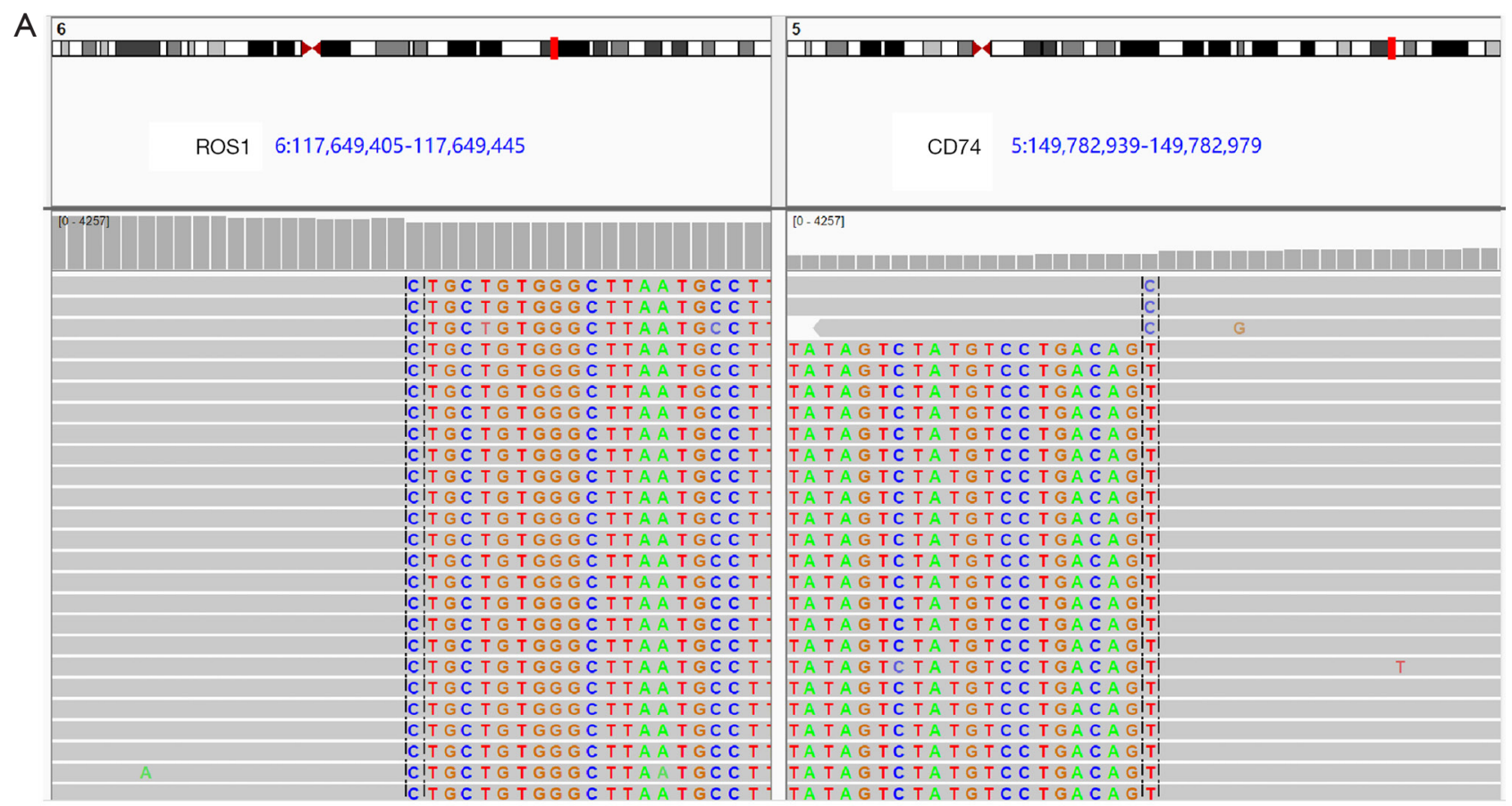

B
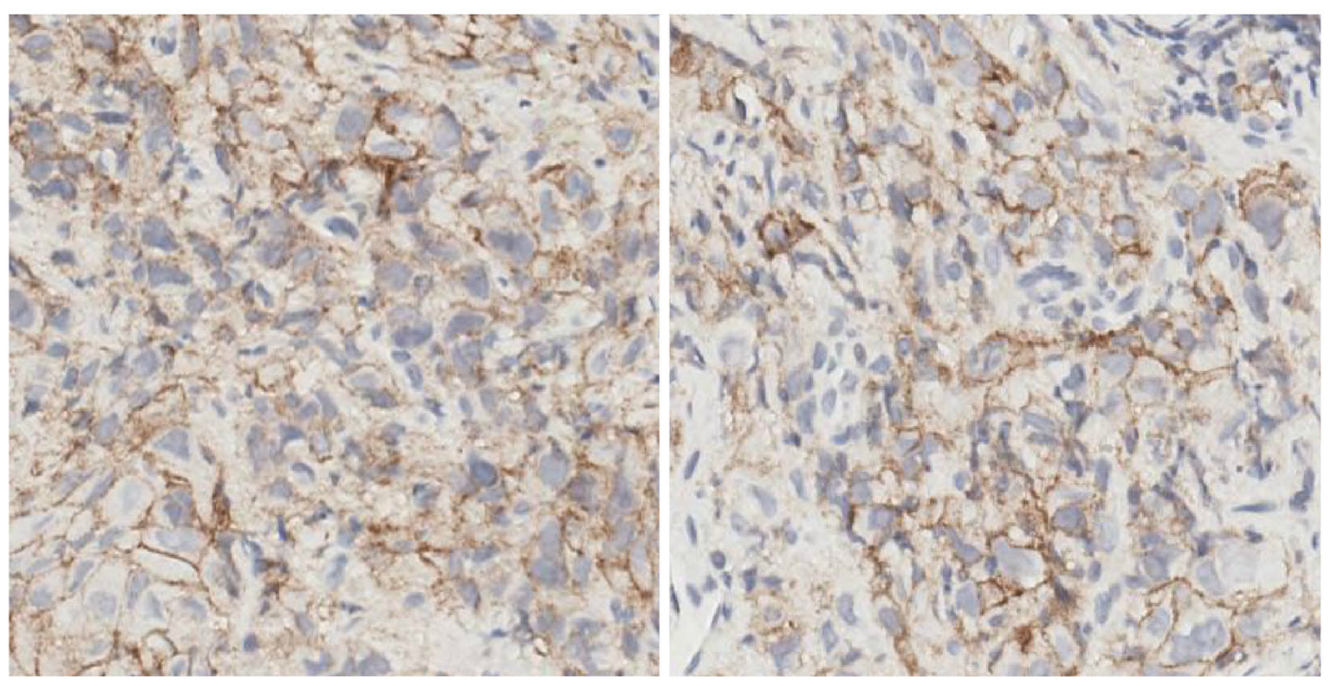

Figure 2 CD74-ROS1 rearrangement and PD-L1 expression detected in the lung biopsy specimen from the patient after crizotinib resistance. (A) The IGV display of CD74-ROS1 rearrangement detected by next-generation sequencing. (B) Representative images of PDL1 IHC staining using 22C3 antibody. ×200 magnification. PD-L1, programmed death-ligand 1; IGV, Integrative Genomics Viewer; IHC, immunohistochemistry.

the clinical efficacy of crizotinib as a second-line therapy in a lung ASC patient with ROS1 fusion who achieved PR after 1 month. However, the patient's disease progressed after a short period of tumor control, attaining a PFS of only 4 months. There has been 1 case of an $A L K$-positive primary lung cancer patient with multiple brain metastases which was temporarily reduced by alectinib, but developed leptomeningeal carcinomatosis after 3 months treatment. The patient was diagnosed with ASC of the lung, and had a higher ratio of the squamous cell carcinoma component to the adenocarcinoma component in the site exhibiting leptomeningeal carcinomatosis than that in the primary 
tumor of the left lung (14). In that case, the existence of tumors with different cell ratios may have in part led to disease progression in a short period of time. Therefore, we hypothesized that in our case the existence of a specific pathological subtype may have also partly led to progression over a short period of time. Roys et al. discussed the molecular mechanisms of crizotinib resistance in ROS1positive NSCLC, and depicted 2 main patterns, namely target alteration and bypass pathway activation (15). They summarized ROS1 point mutations, including G2032R, D2033N, L2026M, S1986Y/F, and L2155S, which may confer resistance to crizotinib. Unluckily, we did not detect any gene mutations other than CD74-ROS1 rearrangement from the biopsy samples after disease progression using a panel covering 520 cancer-related genes. Therefore, we suggest that ASC patients harboring $A L K$ or ROS1 rearrangement should be given priority to receive crizotinib treatment, and follow-up monitoring should be strengthened for disease progression and drug resistance.

Although PD-L1 expression was high in this patient's lung biopsy tissue, the patient did not benefit from albuminbound paclitaxel plus camrelizumab therapy. A retrospective study of patients receiving immune checkpoint inhibitor (ICI) monotherapy for advanced NSCLC with at least 1 oncogenic driver alteration showed that the outcomes of patients treated with ICI monotherapy were consistent with ICI registration trials but were inferior for patients with actionable driver mutations (16). In a phase II study in TKI naïve PD-L1+EGFR-mutant patients with advanced NSCLC, pembrolizumab failed to show efficacy even in patients with PD-L1 expression $\geq 50 \%$ (17). Therefore, the National Comprehensive Cancer Network (NCCN) NSCLC panel recommends that patients with metastatic NSCLC and PD-L1 expression levels of $1 \%$ or more, but who also have a targetable driver oncogene molecular variant (e.g., EGFR, $A L K$, ROS1), should receive first-line targeted therapy for that oncogene and not first-line ICIs. Furthermore, TMB has been proven to be associated with improved survival in patients receiving ICIs across a wide variety of cancer types (18). We assessed the TMB of the biopsy tissues at baseline and after crizotinib treatment, and found that both TMB values were nearly 0 mutation/ $\mathrm{MB}$, which may indicate the lack of tumor neoantigens to stimulate the specific T-cell response.

Our case is the first to report the therapeutic effects of crizotinib and checkpoint inhibitor treatment in a rare ASC patient harboring CD74-ROS1 fusion. However, there are several limitations. Firstly, we did not present the pathological images of the patient's initial diagnosis in our manuscript, as she was diagnosed at another hospital and she passed away in April 2021. Secondly, we failed to explore the molecular mechanisms of crizotinib resistance in this ROS1-fusion ASC patient, as we did not detect any gene mutations other than CD74-ROS1 rearrangement from the biopsy samples after disease progression using a panel of 520 cancer-related genes. More studies are needed to investigate the mechanisms of crizotinib resistance in ROS1-fusion ASC patients.

In summary, this is the first case report demonstrating response to crizotinib in a lung ASC patient with ROS1 fusion, and may aid in future investigations into targeted therapy and prognostic evaluation for patients with rare pathological subtypes of NSCLC.

\section{Acknowledgments}

We owe thanks to the patient and her family. We would also like to thank Wenbo Dong, Ying Sun, Qiaolin Kang, Lihong $\mathrm{Wu}$, and Yujuan Liu from Burning Rock Biotech for their assistance and suggestions in data interpretation and manuscript writing.

Funding: This work was supported by two grants from Science and Technology Program of Tongzhou District, Beijing, China (No. KJ2020CX006-22; No. KJ2020CX004-28).

\section{Footnote}

Reporting Checklist: The authors have completed the CARE reporting checklist. Available at https://atm.amegroups. com/article/view/10.21037/atm-21-6754/rc

Conflicts of Interest: All authors have completed the ICMJE uniform disclosure form (available at https://atm.amegroups. com/article/view/10.21037/atm-21-6754/coif). The authors report that Burning Rock Biotech offered the assistance and suggestions in data interpretation and manuscript writing, but the authors have no financial interest in Burning Rock Biotech. The authors have no other conflicts of interest to declare.

Ethical Statement: The authors are accountable for all aspects of the work in ensuring that questions related to the accuracy or integrity of any part of the work are appropriately investigated and resolved. All procedures performed in this study were in accordance with the ethical 
standards of the institutional and/or national research committee(s) and with the Helsinki Declaration (as revised in 2013). Written informed consent was obtained from the patient for publication of this case report and accompanying images. A copy of the written consent is available for review by the editorial office of this journal.

Open Access Statement: This is an Open Access article distributed in accordance with the Creative Commons Attribution-NonCommercial-NoDerivs 4.0 International License (CC BY-NC-ND 4.0), which permits the noncommercial replication and distribution of the article with the strict proviso that no changes or edits are made and the original work is properly cited (including links to both the formal publication through the relevant DOI and the license). See: https://creativecommons.org/licenses/by-nc-nd/4.0/.

\section{References}

1. Barta JA, Powell CA, Wisnivesky JP. Global Epidemiology of Lung Cancer. Ann Glob Health 2019;85:8.

2. $\mathrm{Li} \mathrm{C}, \mathrm{Lu} \mathrm{H}$. Adenosquamous carcinoma of the lung. Onco Targets Ther 2018;11:4829-35.

3. Filosso PL, Ruffini E, Asioli S, et al. Adenosquamous lung carcinomas: a histologic subtype with poor prognosis. Lung Cancer 2011;74:25-9.

4. Mordant P, Grand B, Cazes A, et al. Adenosquamous carcinoma of the lung: surgical management, pathologic characteristics, and prognostic implications. Ann Thorac Surg 2013;95:1189-95.

5. Chaft JE, Rekhtman N, Ladanyi M, et al. ALK-rearranged lung cancer: adenosquamous lung cancer masquerading as pure squamous carcinoma. J Thorac Oncol 2012;7:768-9.

6. Birchmeier C, Birnbaum D, Waitches G, et al. Characterization of an activated human ros gene. Mol Cell Biol 1986;6:3109-16.

7. Davies KD, Doebele RC. Molecular pathways: ROS1 fusion proteins in cancer. Clin Cancer Res 2013;19:4040-5.

8. Chong CR, Bahcall M, Capelletti M, et al. Identification

Cite this article as: Cheng Y, Yang J, Wang D, Yan D. ROS1 fusion lung adenosquamous carcinoma patient with short-term clinical benefit after crizotinib treatment: a case report. Ann Transl Med 2022;10(3):157. doi: 10.21037/atm-21-6754 of existing drugs that effectively target NTRK1 and ROS1 rearrangements in lung cancer. Clin Cancer Res 2017;23:204-13.

9. Uguen A, De Braekeleer M. ROS1 fusions in cancer: a review. Future Oncol 2016;12:1911-28.

10. Davies KD, Le AT, Theodoro MF, et al. Identifying and targeting ROS1 gene fusions in non-small cell lung cancer. Clin Cancer Res 2012;18:4570-9.

11. Shaw AT, Ou SH, Bang YJ, et al. Crizotinib in ROS1rearranged non-small-cell lung cancer. $\mathrm{N}$ Engl J Med 2014;371:1963-71.

12. Shaw AT, Riely GJ, Bang YJ, et al. Crizotinib in ROS1rearranged advanced non-small-cell lung cancer (NSCLC): updated results, including overall survival, from PROFILE 1001. Ann Oncol 2019;30:1121-6.

13. Solomon BJ, Mok T, Kim DW, et al. First-line crizotinib versus chemotherapy in ALK-positive lung cancer. $\mathrm{N}$ Engl J Med 2014;371:2167-77.

14. Takeda M, Sato K, Sakamoto S, et al. An autopsy case of anaplastic lymphoma kinase-positive lung cancer exacerbated in a short period of time: a case report. J Med Case Rep 2019;13:118.

15. Roys A, Chang X, Liu Y, et al. Resistance mechanisms and potent-targeted therapies of ROS1-positive lung cancer. Cancer Chemother Pharmacol 2019;84:679-88.

16. Mazieres J, Drilon A, Lusque A, et al. Immune checkpoint inhibitors for patients with advanced lung cancer and oncogenic driver alterations: results from the IMMUNOTARGET registry. Ann Oncol 2019;30:1321-8.

17. Lisberg A, Cummings A, Goldman JW, et al. A Phase II Study of Pembrolizumab in EGFR-Mutant, PD-L1+, Tyrosine Kinase Inhibitor Naive Patients With Advanced NSCLC. J Thorac Oncol 2018;13:1138-45.

18. Samstein RM, Lee CH, Shoushtari AN, et al. Tumor mutational load predicts survival after immunotherapy across multiple cancer types. Nat Genet 2019;51:202-6.

(English Language Editor: C. Betlazar-Maseh) 